\title{
Vascular repair and anti-inflammatory effects of soluble epoxide hydrolase inhibitor
}

\author{
NA DAI ${ }^{1,2}$, CUIFEN ZHAO ${ }^{1}$, QINGYU KONG ${ }^{1}$, DONG LI $^{1}$, ZHIFENG CAI $^{1}$ and MINMIN WANG ${ }^{1}$ \\ ${ }^{1}$ Department of Pediatrics, Qilu Hospital, Shandong University; ${ }^{2}$ Department of Pediatrics, \\ Jinan Maternity and Child Care Hospital, Ji'nan, Shandong 250012, P.R. China
}

Received June 9, 2018; Accepted December 13, 2018

DOI: $10.3892 /$ etm.2019.7396

\begin{abstract}
Kawasaki disease (KD) is the leading cause of acquired heart disease in pediatric patients in developed countries. Coronary artery aneurysms and myocardial infarction may occur if the disease remains untreated. An estimated $10-20 \%$ of KD patients do not respond to intravenous gamma globulin (IVIG), and thus, alternative treatments are currently being investigated. Epoxyeicosatrienoic acids (EETs) are natural anti-inflammatory factors and angiogenic mediators degraded by soluble epoxide hydrolase (sEH). sEH inhibitory factors have been demonstrated to stabilize EET levels, inhibit inflammation and promote vascular repair in vivo. The present study aimed to determine whether an increase in EET levels induced by treatment with the sEH inhibitor 12-(3-adamantan-1-yl-ureido)-dodecanoic acid (AUDA) promotes vascular repair in human coronary arterial endothelial cells (HCAECs) and reduces inflammation in a mouse model of KD induced by Lactobacillus casei cell wall extract. The effect of AUDA on vascular repair in HCAECs was assessed by using cell proliferation, migration, adhesion and tube formation assays, and the anti-inflammatory effect of AUDA in the mouse model of KD was determined by detecting the expression of matrix metalloproteinase (MMP)-9, tumor necrosis factor (TNF) $-\alpha$ and interleukin (IL)-1 $\beta$ at the protein level via ELISA. The results demonstrated that AUDA increased the proliferation, migration, adhesion and tube formation ability of HCAECs in a dose-dependent manner. Furthermore, in the mouse model of KD, AUDA reduced the protein expression of MMP-9, IL-1 $\beta$ and TNF- $\alpha$, indicating that AUDA may alleviate inflammatory reactions in the coronary arteries of KD model mice. The present results also indicate that these effects may be exerted through the peroxisome proliferator activated
\end{abstract}

Correspondence to: Dr Cuifen Zhao, Department of Pediatrics, Qilu Hospital, Shandong University, 107 West of Wenhua Road, Ji'nan, Shandong 250012, P.R. China

E-mail: zhaocuifen@sdu.edu.cn

Key words: kawasaki disease, human coronary arterial endothelial cells, soluble epoxide hydrolase, epoxyeicosatrienoic acids, soluble epoxide hydrolase inhibitors receptor $\gamma$ signaling pathway. Taken together, the present study supports the potential utility of AUDA in the treatment of KD.

\section{Introduction}

Kawasaki disease (KD) is an acute and systemic autoimmune vasculitis with unknown etiology, primarily occurring in pediatric patients aged $<5$ years $(1,2)$. It is well known as the leading cause of acquired heart disease in children in developed countries (3). Although the etiology remains elusive, KD contributes to systemic inflammation and has a clear preference for coronary arteries.

The histological manifestations of KD-associated coronary arteritis are inflammatory cell infiltration with breakdown of the extracellular matrix, particularly of the elastic tissue in the vascular media, resulting in coronary artery aneurysm (CAA) formation (4). Mortality in KD is usually caused by ischemic cardiomyopathy (5).

Limited knowledge of the etiologic factors and cell molecular pathology of vasculitis has hampered the discovery of more effective KD treatments or therapies (6-8). At present, single-dose intravenous $\gamma$ globulin (IVIG) is used to effectively reduce the prevalence of CAAs and is the preferred treatment for preventing coronary lesions in pediatric patients with KD (9), but an estimated $10-20 \%$ of patients are not sensitive to this treatment which results in poor prognosis (3). The optimum treatment for IVIG non-responders remains inconclusive, and drugs for secondary or 'rescue' treatment vary by center. Thus, it is important to explore the pathogenesis of KD and identify alternative treatments.

Soluble epoxide hydrolase inhibitors (sEHi) protect the cardiovascular system in multiple ways (10-12), e.g., by inhibiting the deactivation of epoxyeicosatrienoic acids (EETs) and at times by sEH-mediated effects on inflammation-associated diols $(13,14)$. EETs are essential for maintaining the normal function of an organism and may cause significant vasodilation, alleviate inflammation, inhibit the migration of vascular smooth muscle cells and platelet aggregation, promote fibrinolysis and reduce adhesion factor expression (15-17). Furthermore, it has been reported that EETs are involved in vascular repair by inducing angiogenesis (18). Inhibition of sEH increases the positive roles of EETs in atherosclerosis, hypertension, myocardial hypertrophy, ischemic heart disease, diabetes-associated heart failure and metabolic syndrome in vivo (19-24). 
However, it remains elusive whether sEHi have any therapeutic effect in KD. Therefore, the present study aimed to determine whether the sEHi 12-(3-adamantan-1-yl-ureido)-dodecanoic acid (AUDA) promotes the vascular repair of human coronary arterial endothelial cells (HCAECs) and reduces inflammation in the coronary artery in a KD mouse model induced by Lactobacillus casei cell wall extract (LCWE). The present study further sought to reveal the role of the EET/peroxisome proliferator activated receptor $\gamma(\operatorname{PPAR} \gamma)$ pathway in the effect of AUDA on HCAECs and the mouse model of KD. The results suggest a potential role of AUDA in promoting the vascular repair of HCAECs and in alleviating the inflammatory response in $\mathrm{KD}$.

\section{Materials and methods}

Cell culture and treatments. HCAECs were obtained from the Wuhan Culture Collection and maintained in endothelial culture medium (ECM) with 5\% fetal bovine serum (FBS), $1 \%$ penicillin/streptomycin solution and $1 \%$ endothelial cell growth supplement (all from ScienCell Research Laboratories, Inc., San Diego, CA, USA) at $37^{\circ} \mathrm{C}$ in $5 \% \mathrm{CO}_{2}$ in air. HCAECs were treated with different concentrations of $\operatorname{AUDA}(0,1,10$, 50 or $100 \mu \mathrm{mol} / \mathrm{l}$ ) for $24 \mathrm{~h}$.

To further investigate the role of the PPAR $\gamma$ pathway in the role of AUDA in HCAECs, the PPAR $\gamma$ antagonist GW9662 was used. HCAECs were cultured with GW9662 (5 $\mu \mathrm{mol} / \mathrm{l})$ for $30 \mathrm{~min}$, followed by the addition of $100 \mu \mathrm{mol} / 1$ AUDA.

Cell migration assay. For migration assays, 24-well Transwell plates with $8-\mu \mathrm{m}$ pore size and $6.5 \mathrm{~mm}$-diameter polycarbonate filters (Costar; Corning Incorporated, Corning, NY, USA) were used. HCAECs $(100 \mu \mathrm{l})$ were resuspended in serum-free ECM at a density of $1 \times 10^{5}$ cells $/ \mathrm{ml}$ and $100 \mu \mathrm{l}$ was seeded onto the upper chamber, while ECM supplemented with $5 \%$ FBS was added to the lower chamber. Following 24-h culture, the migrated cells were fixed with $4 \%$ paraformaldehyde for 20 min, washed with PBS and stained with $100 \mu 10.1 \%$ crystal violet for $30 \mathrm{~min}$. Quantitative analysis of migrated cells was performed. Cells in 10 randomly selected fields per well were observed and counted under a phase-contrast microscope (magnification, x100; Olympus BH2; Olympus, Tokyo, Japan). Experiments were performed in triplicate.

Cell adhesion assay. At 90\% confluence, HCAECs were seeded into 96-well culture plates coated in fibronectin (BD Biosciences, San Jose, CA, USA) at density of $1 \times 10^{4}$ cells/well and cultured for $1 \mathrm{~h}$ at $37^{\circ} \mathrm{C}$. Following incubation, non-adherent cells were washed with PBS three times, followed by fixation with $4 \%$ paraformaldehyde for $20 \mathrm{~min}$, and staining with $100 \mu 10.1 \%$ crystal violet for $30 \mathrm{~min}$. Adherent cells in 10 randomly selected fields per well were observed and counted under a phase-contrast microscope (magnification, x100; Olympus BH2; Olympus). Experiments were performed in triplicate.

Capillary-like tube formation assay. Matrigel ${ }^{\circledR}$ (BD Biosciences) was thawed on ice overnight and once thawed, $50 \mu \mathrm{l}$ was added to each well of a 96-well plate and incubated for $1 \mathrm{~h}$ at $37^{\circ} \mathrm{C}$ to solidify. HCAECs were seeded into 96 -well plates pre-coated with Matrigel ${ }^{\circledR}$ at a density of $1 \times 10^{4}$ cells/well and incubated at $37^{\circ} \mathrm{C}$ for $6 \mathrm{~h}$. Images of tube formation were captured using an inverted light microscope (magnification, x100; Olympus BH2; Olympus). Segment lengths were measured using Image J software (version $1.44 \mathrm{p}$; National Institutes of Health, Bethesda, MD, USA) in 5 randomly selected fields per well and the average segment length per field was calculated.

Cell proliferation assay. Cell counting kit-8 (CCK-8; Beyotime Institute of Biotechnology, Haimen, China) was used to determine the proliferation of HCAECs following the manufacturer's protocol. The CCK- 8 assay utilizes the yellow formazan dye produced following the reduction of tetrazolium salt WST- 8 by the mitochondria of live cells to determine cell activity.

In brief, $2 \times 10^{3}$ cells in $100 \mu \mathrm{l}$ medium were added to each well of a 96-well plate and cultured overnight. Cells were subsequently treated with various concentrations $(0,1,10,50$ or $100 \mu \mathrm{mol} / \mathrm{l}$ ) of AUDA for $24 \mathrm{~h}$. Following incubation, $10 \mu \mathrm{l}$ CCK-8 solution was added and cells were incubated at $37^{\circ} \mathrm{C}$ for a further $4 \mathrm{~h}$. The optical density was measured at $490 \mathrm{~mm}$ using a microplate reader (Bio-Rad Laboratories, Hercules, CA, USA).

LCWE. LCWE was prepared as previously described (25). The concentration of LCWE (in PBS) was measured by analyzing the rhamnose content determined via a phenol-sulfuric acid colorimetric assay.

Mice. In total 20, male (wild-type) C57BL/6 mice (age, 4-6 weeks; weight, 18-20 g) were obtained from the Animal Centre of Shandong Medical University (Shandong, China). All mice were maintained under specific pathogen-free conditions $\left(20-26^{\circ} \mathrm{C}, 40-70 \%\right.$ humidity, 12-h light/dark cycle with access to full-valence granular rat feedstuff and sterile water ad libitum). Mice were randomly divided into four groups: PBS, LCWE, LCWE+AUDA and LCWE+AUDA+GW9662. In each group, mice were injected intraperitoneally with $0.5 \mathrm{ml}$ PBS alone; PBS supplemented with $0.5 \mathrm{mg}$ LCWE; PBS supplemented with $0.5 \mathrm{mg}$ LCWE and $10 \mathrm{mg} / \mathrm{kg}$ AUDA (Cayman Chemical, Wuhan, China); or PBS supplemented with $0.5 \mathrm{mg} \mathrm{LCWE}, 10 \mathrm{mg} / \mathrm{kg}$ AUDA and $10 \mathrm{mg} / \mathrm{kg}$ GW9662 (MedchemExpress, Shanghai, China), respectively. Following 14-day induction, mice were sacrificed.

ELISA. The supernatant of HCAECs and total protein of murine hearts were used for the detection of matrix metallopeptidase (MMP)-9, interleukin (IL)-1 $\beta$ and tumor necrosis factor (TNF)- $\alpha$ by means of ELISA. Lysis buffer (Lichen, Shanghai, China) was used for the homogenization of murine hearts, and total protein was extracted following the manufacturer's instructions. Protein levels of MMP-9, IL-1 $\beta$ and TNF- $\alpha$ were examined using ELISA kits (MMP-9, cat. no. TY02784B; IL-1 $\beta$, cat. no., lc-005; TNF- $\alpha$, cat. no. lc-007; all Yingxin Laboratory Equipment Co., Ltd., Shanghai, China).

Statistical analysis. Prism 5.0 (GraphPad Software, Inc., La Jolla, CA, USA) was used for data analysis. Data are expressed 

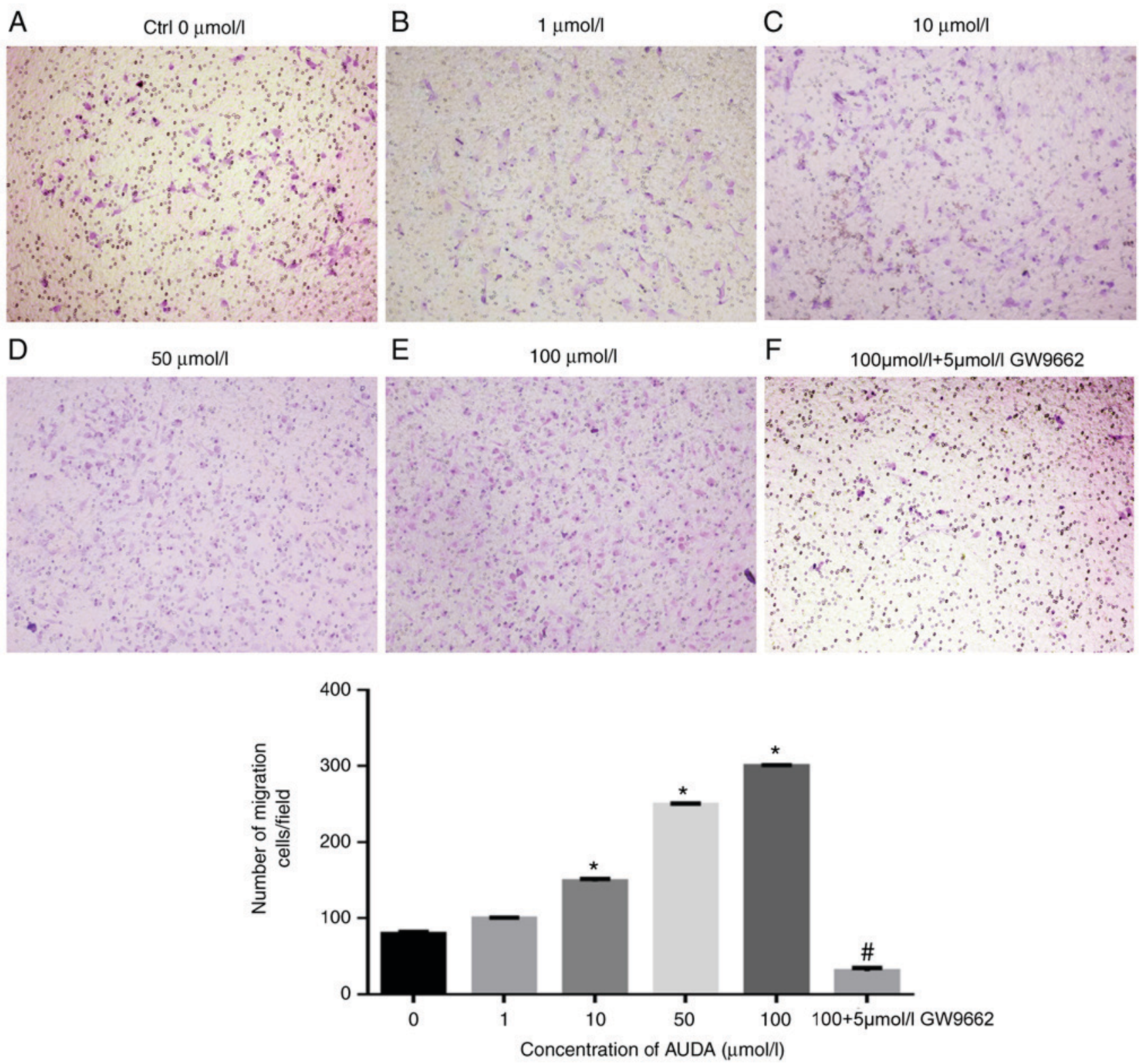

Figure 1. Effect of AUDA on HCAEC migration. (A-E) Migration of HCAECs treated with 0, 1, 10, 50 or $100 \mu$ mol/1 AUDA. (F) Migration of HCAECs treated with $100 \mu \mathrm{mol} / 1$ AUDA $+5 \mu \mathrm{mol} / 1 \mathrm{GW} 9662$. The number of migrated HCAECs per 10 randomly selected fields per well were observed and counted. Representative photomicrographs are provided at a magnification of $\mathrm{x} 100$. Values are expressed as the mean \pm standard error of the mean $(\mathrm{n}=10)$. ${ }^{*} \mathrm{P}<0.05 \mathrm{vs}$. Ctrl ( $0 \mu \mathrm{mol} / 1)$; ${ }^{*} \mathrm{P}<0.05$ vs. $100 \mu \mathrm{mol} / 1$ AUDA. AUDA, 12-(3-adamantan-1-yl-ureido)-dodecanoic acid, an inhibitor of soluble epoxide hydrolase; HCAECs, human coronary arterial endothelial cells; Ctrl, control.

as the mean \pm standard error. The significance of differences among several groups was determined using one-way analysis of variance with Bonferroni correction. $\mathrm{P}<0.05$ was considered to indicate a statistically significant difference.

\section{Results}

Effect of AUDA on the migration of HCAECs. As presented in Fig. 1, AUDA augmented the migratory ability of HCAECs in a dose-dependent manner. Compared with that in the control group $(0 \mu \mathrm{mol} / 1$ AUDA), treatment with 10,50 and $100 \mu \mathrm{mol} / 1$ AUDA resulted in a significant increase in HCAEC migration $(\mathrm{P}<0.05)$. To further investigate the effect of AUDA on HCAECs, the PPAR $\gamma$ antagonist GW9662 was used to examine the PPAR $\gamma$ signaling pathway. Following pre-treatment with
GW9662 $(5 \mu \mathrm{mol} / \mathrm{l})$, the AUDA-induced increase in HCAEC migration was significantly suppressed $(\mathrm{P}<0.05)$.

Effect of AUDA on the adhesion of HCAECs. Next, the effect of AUDA on the adhesion of HCAECs was evaluated, revealing that AUDA increased cell adhesion in a dose-dependent manner. Compared with that in the control group $(0 \mu \mathrm{mol} / 1$ AUDA), 10, 50 and $100 \mu \mathrm{mol} / 1$ AUDA significantly increased the adhesion ability of HCAECs $(\mathrm{P}<0.05)$. However, cell adhesion was severely impaired by pre-treatment with GW9662 followed by AUDA (P<0.05; Fig. 2).

Effect of AUDA on in vitro angiogenesis in HCAECs. The effect of AUDA on capillary tube formation was then evaluated as a measure of in vitro angiogenesis in HCAECs. AUDA 

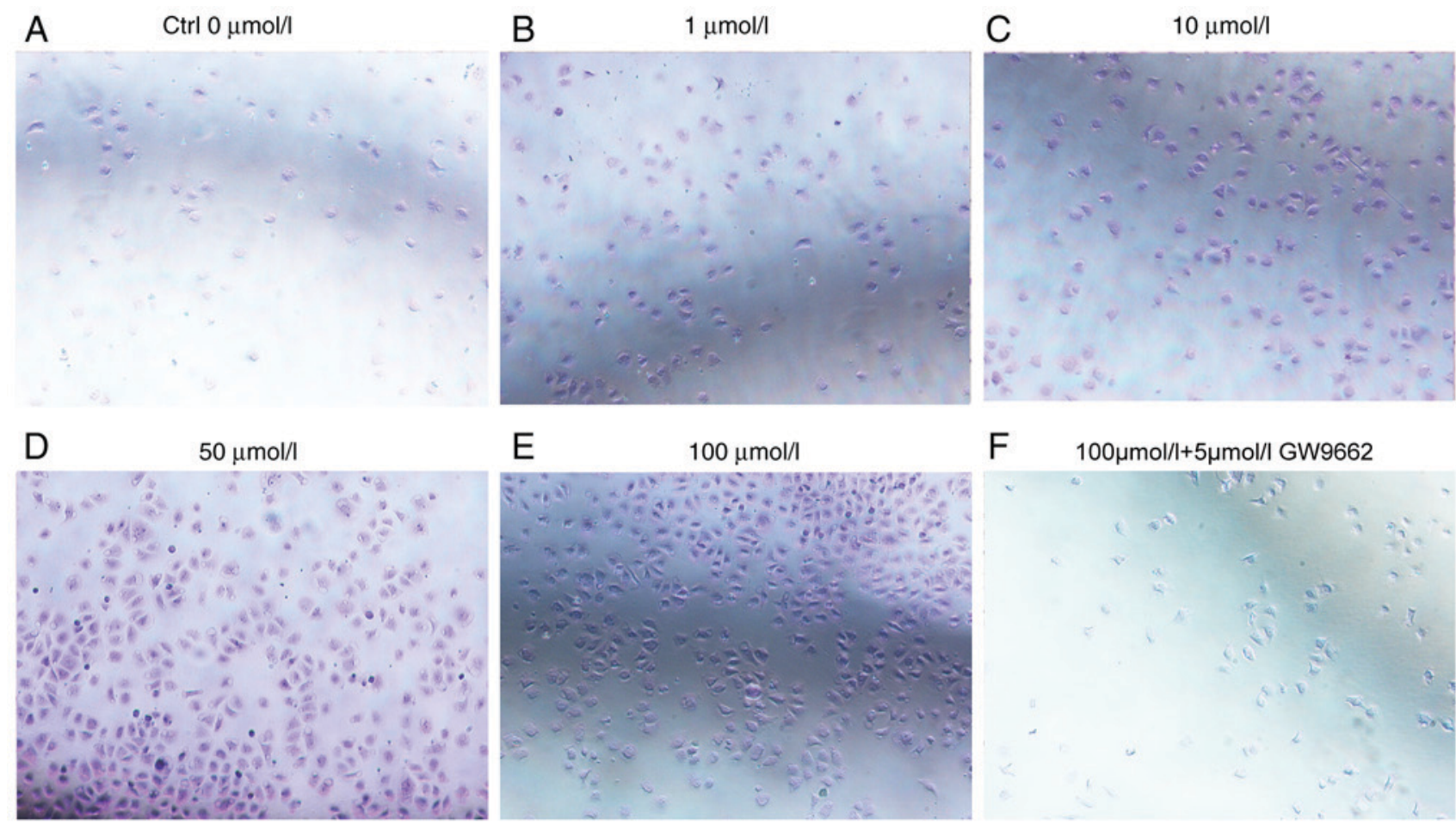

F $\quad 100 \mu \mathrm{mol} / 1+5 \mu \mathrm{mol} / \mathrm{G}$ GW962

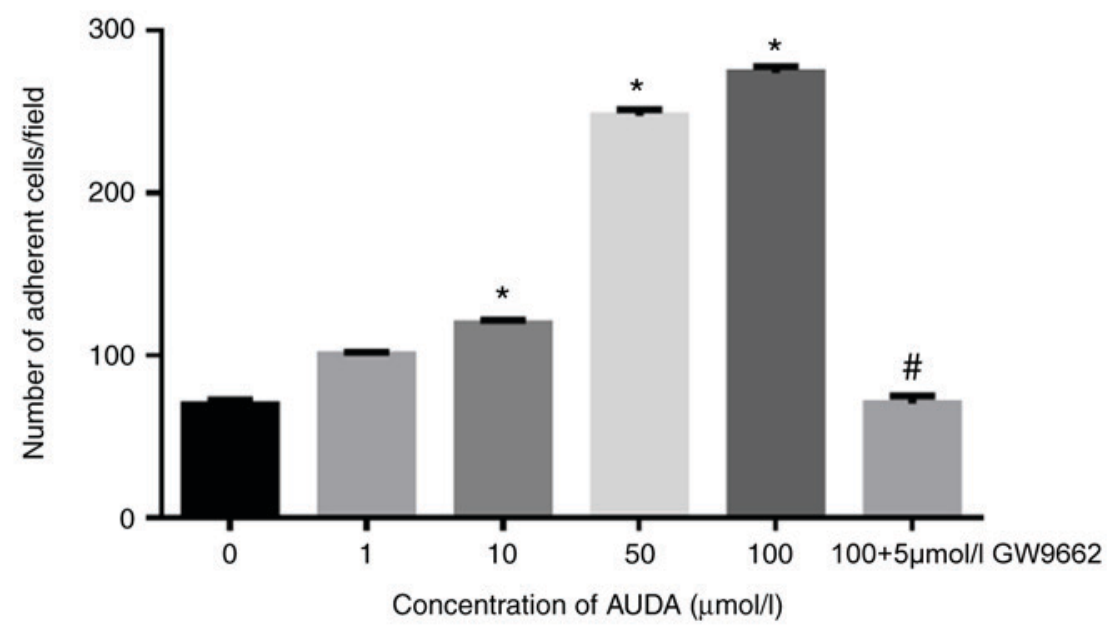

Figure 2. Effect of AUDA on HCAEC adhesion. (A-E) Adhesion of HCAECs treated with 0,1,10,50 or $100 \mu$ mol/1 AUDA. (F) Adhesion of HCAECs treated with $100 \mu \mathrm{mol} / 1$ AUDA $+5 \mu \mathrm{mol} / 1$ GW9662. The number of adherent HCAECs per 10 randomly selected fields per well were observed and counted. Representative photomicrographs are provided at a magnification of $\mathrm{x} 100$. Values are expressed as the mean \pm standard error of the mean $(\mathrm{n}=10)$. ${ }^{*} \mathrm{P}<0.05 \mathrm{vs}$. Ctrl (0 $\mu \mathrm{mol} / \mathrm{l}) ;{ }^{*} \mathrm{P}<0.05$ vs. $100 \mu \mathrm{mol} / 1$ AUDA. AUDA, 12-(3-adamantan-1-yl-ureido)-dodecanoic acid, an inhibitor of soluble epoxide hydrolase; HCAECs, human coronary arterial endothelial cells; Ctrl, control.

increased the capillary tube formation of HCAECs in vitro in a dose-dependent manner (Fig. 3). In comparison with that observed in the control group ( $0 \mu \mathrm{mol} / 1$ AUDA), 1,10 , 50 and $100 \mu \mathrm{mol} / 1$ AUDA markedly promoted the capillary tube formation ability of HCAECs in vitro $(\mathrm{P}<0.05)$, while pre-treatment with GW9662 followed by AUDA significantly blocked tube formation ( $\mathrm{P}<0.05$; Fig. 3$)$.

Effect of AUDA on the proliferation of HCAECs. To further explore the role of AUDA in the proliferation of HCAECs, HCAECs were treated with different concentrations of AUDA $(1,10,50$ and $100 \mu \mathrm{mol} / \mathrm{l})$ and the cell proliferation was determined using the CCK- 8 assay. The results presented in Fig. 4A indicate that AUDA increased the proliferation of HCAECs in a dose-dependent manner. Compared with that in the control group ( $0 \mu \mathrm{mol} / 1$ AUDA), $1,10,50$ and $100 \mu \mathrm{mol} / 1$ AUDA significantly increased the proliferation of HCAECs $(\mathrm{P}<0.05)$. However, the cell proliferation was severely attenuated by pre-treatment with GW9662 (P<0.05; Fig. 4A).

Effects of AUDA on the PPAR $\gamma$ signaling pathway of HCAECs. To further confirm the effect of AUDA on the PPAR $\gamma$ signaling pathway, the proliferation of HCAECs treated with $100 \mu \mathrm{mol} / 1$ AUDA combined with different concentrations of GW9662 was detected. As presented in Fig. 4B, the cell proliferation was attenuated by pre-treatment with GW9662 in a dose-dependent manner. The expression of the inflammatory factors TNF- $\alpha$, IL- $1 \beta$ and MMP-9 in HCAECs was 


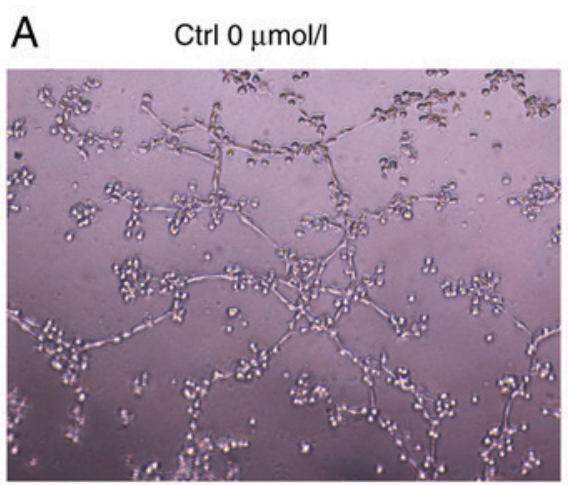

B $\quad 1 \mu \mathrm{mol} / \mathrm{l}$

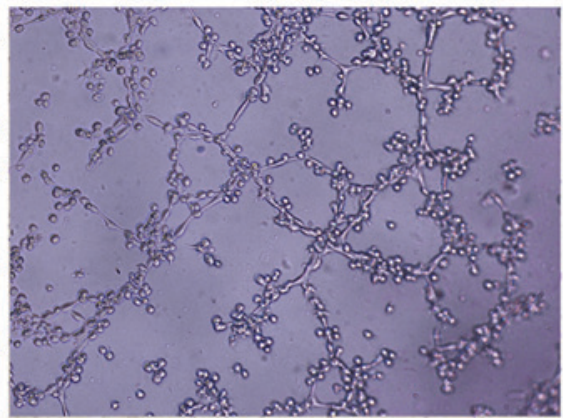

D $\quad 50 \mu \mathrm{mol} / \mathrm{l}$

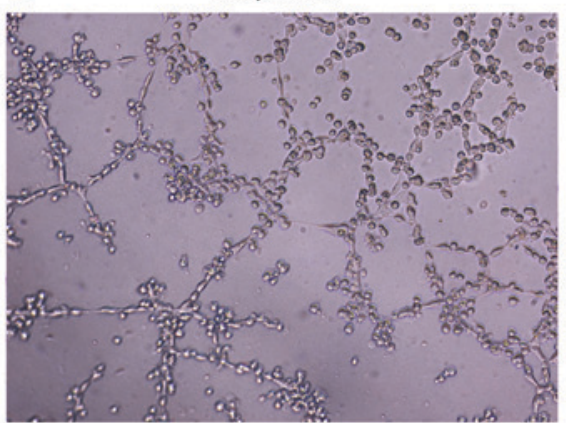

E

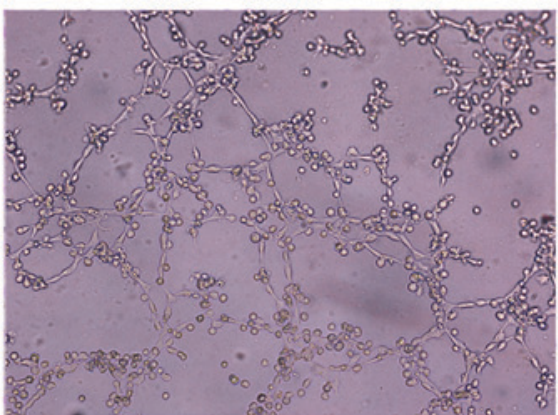

C

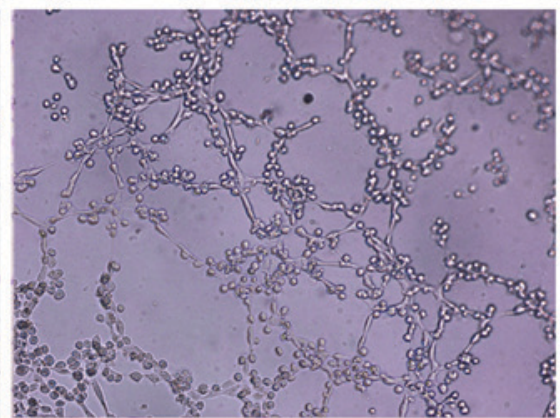

F $\quad 100 \mu \mathrm{mol} / \mathrm{l}+5 \mu \mathrm{mol} / \mathrm{l} \mathrm{GW} 9662$

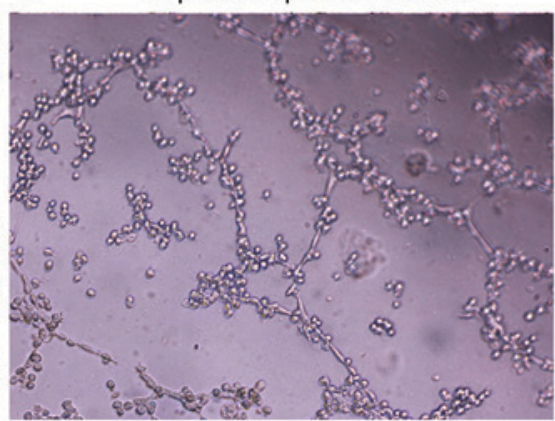

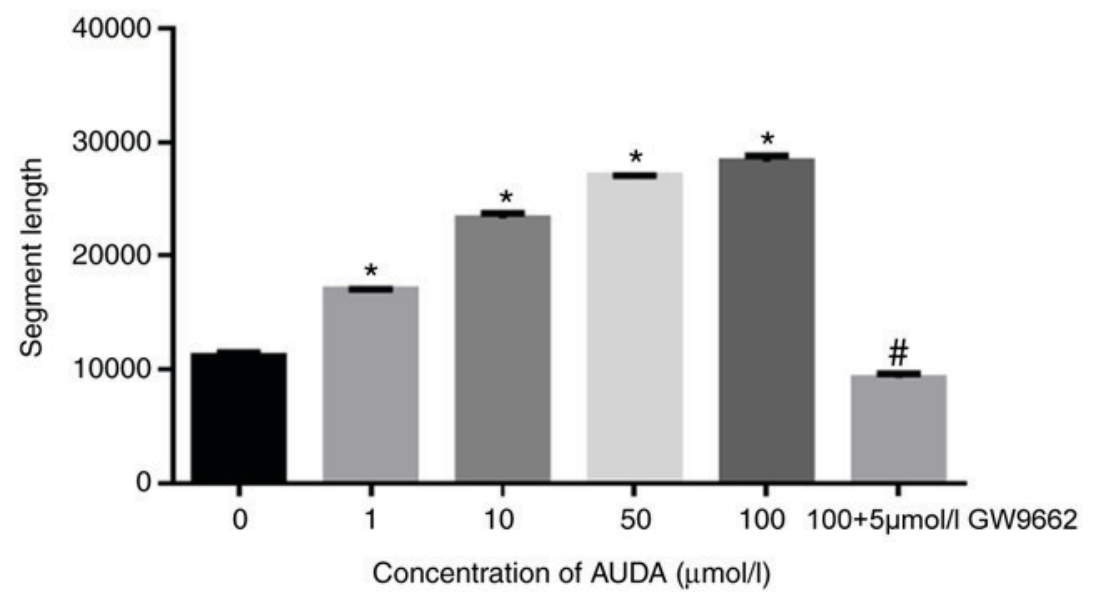

Figure 3. Effect of AUDA on capillary tube formation of HCAECs. (A-E) Tube formation of HCAECs treated with 0, 1, 10, 50 or 100 $\mu$ mol/1 AUDA. (F) Tube formation of HCAECs treated with $100 \mu \mathrm{mol} / 1$ AUDA+ $5 \mu \mathrm{mol} / 1 \mathrm{GW} 9662$. Segment lengths were analyzed in 5 randomly selected high-power fields per sample using Image J software. Representative photomicrographs are provided at a magnification of x100. Values are expressed as the mean \pm standard error of the mean ( $\mathrm{n}=5)$. ${ }^{*} \mathrm{P}<0.05$ vs. Ctrl $(0 \mu \mathrm{mol} / 1)$; ${ }^{\#} \mathrm{P}<0.05$ vs. $100 \mu \mathrm{mol} / 1$ AUDA. AUDA, 12-(3-adamantan-1-yl-ureido)-dodecanoic acid, an inhibitor of soluble epoxide hydrolase; HCAECs, human coronary arterial endothelial cells; Ctrl, control.

then determined using ELISA. The results proved that AUDA inhibited the expression of TNF- $\alpha$, IL-1 $\beta$ and MMP-9 in a dose-dependent manner (Fig. 4C-E). In comparison to the control group ( $0 \mu \mathrm{mol} / 1$ AUDA), 10, 50 and $100 \mu \mathrm{mol} / 1$ AUDA significantly inhibited the expression of inflammatory factors in HCAECs $(\mathrm{P}<0.05)$. However, the inhibitory effect of AUDA on the inflammatory factors was abrogated by pre-treatment with GW9662 followed by AUDA ( $\mathrm{P}<0.05$; Fig. 4C-E).

Inflammation in mouse cardiac tissues. To elucidate the potential role of AUDA in vivo, a KD mouse model was induced by injection of LCWE. Next, the inflammatory responses in the heart tissues of different groups of mice, as determined by examination of the protein expression of the inflammatory factors TNF- $\alpha$, IL-1 $\beta$ and MMP-9, were assessed by ELISA at
14 days after drug injection. As presented in Fig. 5, the relative protein expression of TNF- $\alpha$, IL- $1 \beta$ and MMP-9 was significantly higher in the hearts of mice injected with LCWE than in those injected with PBS alone $(\mathrm{P}<0.05)$. In the AUDA+LCWE injection group, TNF- $\alpha$, MMP-9 and IL-1 $\beta$ expression levels were lower than those in the LCWE group $(\mathrm{P}<0.05)$, while pre-treatment with GW9662 abrogated the effect of AUDA $(\mathrm{P}<0.05)$.

\section{Discussion}

KD is a systemic vasculitis of unknown origin, frequently occurring in pediatric patients. Coronary artery lesions, particularly giant CAAs and severe myocarditis, are potentially fatal complications of KD (26); therefore, the development of 
A
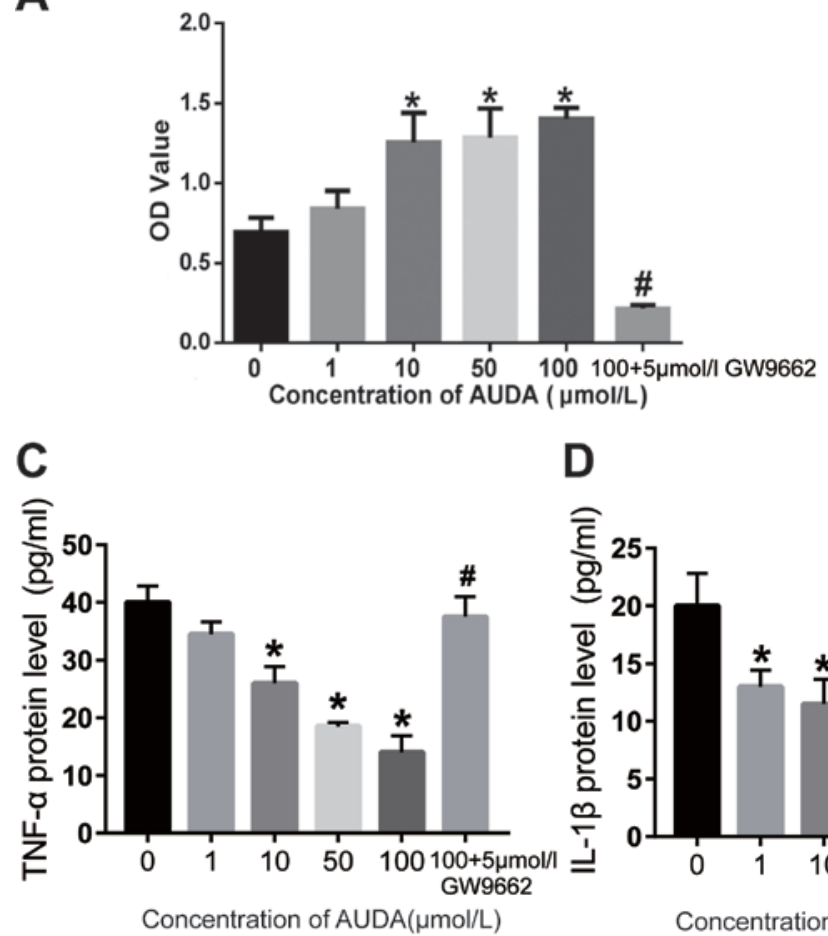

B

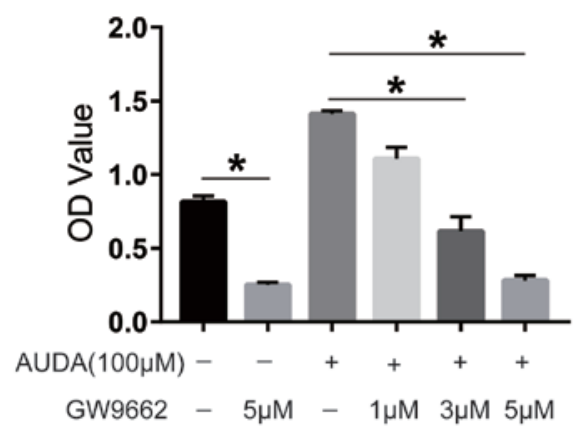

.
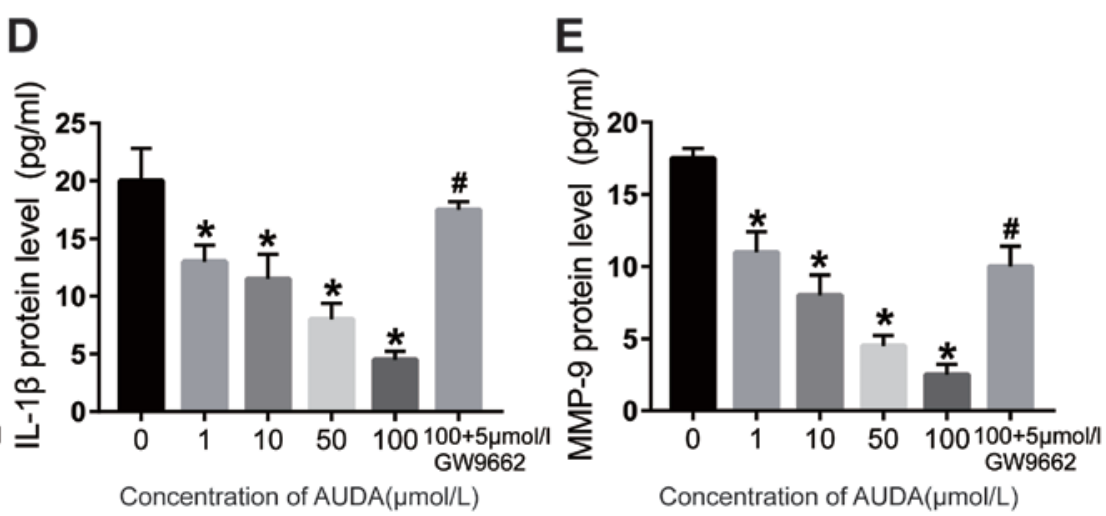

Figure 4. Effect of AUDA on HCAEC proliferation. Cell proliferation was determined using a Cell Counting Kit-8 assay and OD values at $490 \mathrm{~nm}$ are presented. (A) Proliferation of HCAECs treated with 0, 10,50 or $100 \mu \mathrm{mol} / 1$ AUDA with or without $5 \mu \mathrm{mol} / 1$ GW9662. (B) Proliferation of HCAECs treated with $100 \mu \mathrm{mol} / 1$ AUDA combined with different concentrations (0, 1, 3 or $5 \mu \mathrm{M})$ of GW9662. Expression levels of (C) TNF- $\alpha$, (D) IL-1 $\beta$ and (E) MMP-9 were detected by ELISA. Values are expressed as the mean \pm standard error of the mean ( $\mathrm{n}=3 / \mathrm{group})$. ${ }^{\mathrm{P}} \mathrm{P}<0.05 \mathrm{vs}$. Ctrl $(0 \mu \mathrm{mol} / 1)$; $\mathrm{P}<0.05 \mathrm{vs}$. $100 \mu \mathrm{mol} / 1 \mathrm{AUDA}$ OD, optical density; AUDA, 12-(3-adamantan-1-yl-ureido)-dodecanoic acid, an inhibitor of soluble epoxide hydrolase; HCAECs, human coronary arterial endothelial cells; MMP, matrix metallopeptidase; IL, interleukin; TNF, tumor necrosis factor.
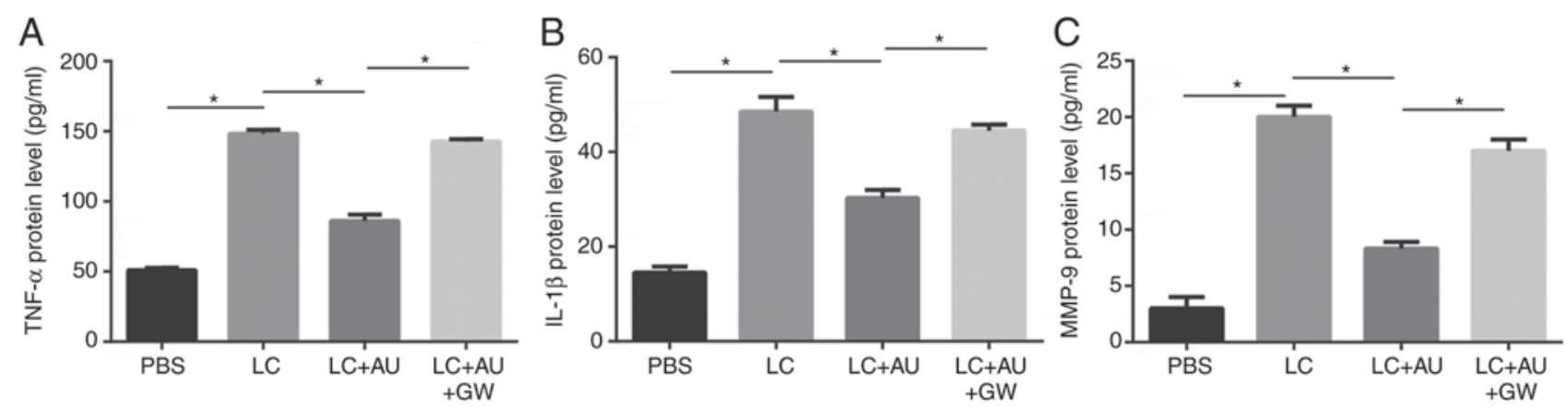

Figure 5. Quantitative analysis of TNF- $\alpha$, IL-1 $\beta$ and MMP-9 protein expression. (A) TNF- $\alpha$, (B) IL-1 $\beta$ and (C) MMP-9 protein expression levels were detected by ELISA in the heart tissue samples of mice in different groups. Values are expressed as the mean \pm standard error of the mean (n=3/group). ${ }^{*} \mathrm{P}<0.05$. Groups: PBS, HCAECs treated with PBS; LC, HCAECs treated with LCWE; LC+AU, HCAECs treated with LCWE and AUDA; LC+AU+GW, HCAECs treated with LCWE, AUDA and GW9662. MMP, matrix metallopeptidase; IL, interleukin; TNF, tumor necrosis factor; AUDA, 12-(3-adamantan-1-yl-ureido)-dodecanoic acid, an inhibitor of soluble epoxide hydrolase; LCWE, Lactobacillus casei cell wall extract.

effective therapies is important for improving the outcome for KD patients. The results of the present study demonstrated the utility of the sEHi AUDA in improving the vascular repair of HCAECs and reduce inflammatory reactions in the coronary artery in KD. The present results also indicated that these effects are dependent on PPAR $\gamma$.

KD causes systemic inflammation and has a clear preference for coronary arteries (27). Coronary arteritis in KD with characteristics of inflammatory cell infiltration and the destruction of elastic tissue in the vascular media results in the occurrence of CAAs (4). EETs have multiple biological functions in normal and pathophysiological processes, exerting anti-inflammatory $(28,29)$, anti-fibrotic (30), anti-apoptotic (31) and anti-oxidant (32) effects. Furthermore, EETs are known to have anti-inflammatory effects on blood vessels (33). It is therefore suggested that EETs have an immunomodulatory effect with potential clinical applications in chronic inflammatory diseases.

However, EETs are rapidly degraded to dihydroxyeicosatrienoic acids with low activity in vivo (13). There is evidence that the suppression of sEH increases EET levels, which represents a possible strategy for improving the biological 
activity of EETs (34). In the present study, the efficacy of the sEHi AUDA in modulating the inflammatory response was investigated.

To further study the anti-inflammatory role of AUDA in $\mathrm{KD}$, a mouse model of KD was employed, and the protein expression levels of IL-1 $\beta$, TNF- $\alpha$ and MMP-9 were measured following AUDA treatment. There is evidence that the expression of TNF- $\alpha$, IL-1 $\beta$ and MMP-9 is associated with the degree of inflammatory infiltrates in the coronary artery walls of LCWE-injected mice (35). Circulating TNF- $\alpha$ contributes to the pathogenesis of coronary artery inflammation as a pivotal pro-inflammatory cytokine, and its expression is significantly upregulated during the KD-associated inflammatory response in a mouse model (36). There is also evidence that the expression of TNF- $\alpha$ in the coronary artery results in the upregulation of MMP-9 in vascular smooth muscle cells, as well as localized electrolytic activity and the matrix destruction of surrounding coronary arteries $(37,38)$. In addition, it has been indicated that the serum levels of IL-1 $\beta$ are markedly increased in KD patients in comparison to those in age-matched healthy controls (39). In view of this, the protein expression levels of TNF- $\alpha$, IL-1 $\beta$ and MMP-9 were examined in the hearts of KD model mice treated with AUDA. The results demonstrated that the protein expression levels were reduced in the mouse model of KD following AUDA treatment, which suggests that AUDA may reduce heart inflammation, and thereby serve a potential role in the therapeutic treatment of KD.

Certain subgroups of KD have a risk of myocardial ischemia from coronary artery thrombosis and stenosis (3). A previous study indicated that EETs promote the vascular repair of endothelial cells via potent pro-mitogenic, pro-migratory and pro-angiogenic effects (40-42). In the present study, it was hypothesized that EETs accelerate the recovery of coronary arteries by promoting the proliferation, migration, adhesion and tube formation ability of HCAECs. To verify this hypothesis, the role of the sEHi AUDA on cell proliferation, migration, adhesion and in vitro angiogenesis of HCAECs was examined. The results of the current study demonstrated that in HCAECs, AUDA promoted cell adhesion, migration, proliferation and tube formation in a dose-dependent manner. AUDA promoted the migration and adhesion of HCAECs. These results suggest that AUDA may promote cell migration and adhesion in HCAECs through interaction with cell surface receptors, leading to cytoskeletal rearrangement, which can serve as a scaffold for cascades of signal transducing molecules (43). However, this hypothesis requires further verification. Taken together, these results suggest that AUDA may be involved in promoting coronary artery recovery. PPAR $\gamma$ activation has been reported to involve the anti-inflammatory functions of EETs (44). PPARs belong to the nuclear receptor superfamily and act as ligand-activated transcription factors. PPARs include three subtypes: PPARa, $\mathrm{PPARb} / \delta$ and PPAR $\gamma$. PPAR $\gamma$ is overexpressed in the skeletal muscle, liver, vascular wall, kidney and heart. PPAR activators have an anti-inflammatory role in a variety of cells through suppressing the levels of pro-inflammatory genes. These results suggest that PPARs have a regulatory role in inflammation and have potential therapeutic applications for chronic inflammatory diseases (45). In addition, a previous study has indicated that EETs act as ligands and endogenous activators for PPAR $\gamma$ (46). Thus, it was speculated that the EET/PPAR $\gamma$ pathway may be responsible for the function of AUDA on HCAECs. The present results indicated that AUDA enhanced HCAEC adhesion and migration in a dose-dependent manner and, this AUDA-induced effect was eliminated following treatment with GW9662, a PPAR $\gamma$ ligand antagonist. These results suggest that AUDA may be involved in promoting metastasis and adhesion by regulating the PPAR $\gamma$ pathway. Furthermore, in the KD mouse model, GW9662 markedly enhanced the protein levels of TNF- $\alpha$, IL-1 $\beta$ and MMP-9. Taken together, the results of the present study suggest that AUDA may exert its angiogenic and anti-inflammatory effects via the EET/PPAR $\gamma$ pathway. In brief, the present study suggests that EETs may act in a PPAR $\gamma$-dependent manner.

In conclusion, the present study investigated the role of AUDA in HCAECs and a mouse model of KD. The results demonstrated that treatment with AUDA reduced the protein expression levels of TNF- $\alpha$, MMP-9 and IL- $1 \beta$ in the KD mouse model and that the vascular repair by HCAECs was markedly increased. These results suggest that AUDA positively modulates the vascular repair function of HCAECs in vitro and alleviates inflammation in heart tissue, demonstrating AUDA as a potential therapeutic treatment of KD.

\section{Acknowledgements}

Not applicable.

\section{Funding}

The present study was supported by grants from the National Natural Science Foundation (grant no. 30900730), the Technology Development Plan of Shandong Province (grant no. 2014GSF118066), the Shandong Province Natural Science Foundation (grant nos. Y2008C44 and Z2008C14) and the Shandong Province Foundation for Excellent Young and Midlife Scholars (grant no. 2005BS02003).

\section{Availability of data and materials}

The datasets used and/or analyzed during the present study are available from the corresponding author on reasonable request.

\section{Authors' contributions}

ND designed the experiments and prepared the manuscript. QK, DL, ZC and MW analyzed and interpreted the data. CZ revised the manuscript and provided technical assistance and advice. All authors read and approved the final manuscript.

\section{Ethical approval and consent to participate}

All animal experiments were performed in accordance with the protocol approved by the Animal Care Committee of Shandong University (Ji'nan, China).

\section{Patient consent for publication}

Not applicable. 


\section{Competing interests}

The authors declare that they have no competing interests.

\section{References}

1. Greco A, De Virgilio A, Rizzo MI, Tombolini M, Gallo A, Fusconi M, Ruoppolo G, Pagliuca G, Martellucci S and de Vincentiis M: Kawasaki disease: An evolving paradigm. Autoimmun Rev 14: 703-709, 2015.

2. Fimbres AM and Shulman ST: Kawasaki disease. Pediatr Rev 29: 308-316, 2008

3. McCrindle BW, Rowley AH, Newburger JW, Burns JC, Bolger AF, Gewitz M, Baker AL, Jackson MA, Takahashi M, Shah PB, et al: Diagnosis, treatment, and long-term management of kawasaki disease: A scientific statement for health professionals from the american heart association. Circulation 135 e927-e999, 2017.

4. Kato H, Sugimura T, Akagi T, Sato N, Hashino K, Maeno Y, Kazue T, Eto $\mathrm{G}$ and Yamakawa R: Long-term consequences of Kawasaki disease. A 10-to 21-year follow-up study of 594 patients. Circulation 94: 1379-1385, 1996.

5. Burns JC, Shike H, Gordon JB, Malhotra A, Schoenwetter M and Kawasaki T: Sequelae of Kawasaki disease in adolescents and young adults. J Am Coll Cardiol 28: 253-257, 1996.

6. Rowley AH, Baker SC, Orenstein JM and Shulman ST: Searching for the cause of Kawasaki disease-cytoplasmic inclusion bodies provide new insight. Nat Rev Microbiol 6: 394-401, 2008.

7. Rowley AH, Baker SC, Shulman ST, Garcia FL, Fox LM, Kos IM, Crawford SE, Russo PA, Hammadeh R, Takahashi K and Orenstein JM: RNA-containing cytoplasmic inclusion bodies in ciliated bronchial epithelium months to years after acute Kawasaki disease. PLoS One 3: e1582, 2008.

8. Rowley AH, Shulman ST, Garcia FL, Guzman-Cottrill JA, Miura M, Lee HL and Baker SC: Cloning the arterial IgA antibody response during acute Kawasaki disease. J Immunol 175 : 8386-8391, 2005.

9. Newburger JW, Takahashi M, Beiser AS, Burns JC, Bastian J, Chung KJ, Colan SD, Duffy CE, Fulton DR, Glode MP, et al: A single intravenous infusion of gamma globulin as compared with four infusions in the treatment of acute Kawasaki syndrome. $\mathrm{N}$ Engl J Med 324: 1633-1639, 1991.

10. Ingraham RH, Gless RD and Lo HY: Soluble epoxide hydrolase inhibitors and their potential for treatment of multiple pathologic conditions. Curr Med Chem 18: 587-603, 2011.

11. Simpkins AN, Rudic RD, Roy S, Tsai HJ, Hammock BD and Imig JD: Soluble epoxide hydrolase inhibition modulates vascular remodeling. Am J Physiol Heart Circ Physiol 298: H795-H806, 2010.

12. Zhang LN, Vincelette J, Cheng Y, Mehra U, Chen D, Anandan SK, Gless R, Webb HK and Wang YX: Inhibition of soluble epoxide hydrolase attenuated atherosclerosis, abdominal aortic aneurysm formation, and dyslipidemia. Arterioscler Thromb Vasc Biol 29: 1265-1270, 2009.

13. Spector AA, Fang X, Snyder GD and Weintraub NL: Epoxyeicosatrienoic acids (EETs): Metabolism and biochemical function. Prog Lipid Res 43: 55-90, 2004.

14. Zeldin DC, Kobayashi J, Falck JR, Winder BS, Hammock BD, Snapper JR and Capdevila JH: Regio- and enantiofacial selectivity of epoxyeicosatrienoic acid hydration by cytosolic epoxide hydrolase. J Biol Chem 268: 6402-6407, 1993.

15. Spector AA and Norris AW: Action of epoxyeicosatrienoic acids on cellular function. Am J Physiol Cell Physiol 292: C996-C1012, 2007.

16. Newman JW, Morisseau C and Hammock BD: Epoxide hydrolases: Their roles and interactions with lipid metabolism. Prog Lipid Res 44: 1-51, 2005.

17. Miller AW, Dimitropoulou C, Han G, White RE, Busija DW and Carrier GO: Epoxyeicosatrienoic acid-induced relaxation is impaired in insulin resistance. Am J Physiol Heart Circ Physiol 281: H1524-H1531, 2001.

18. Bellien J, Joannides R, Richard V and Thuillez C: Modulation of cytochrome-derived epoxyeicosatrienoic acids pathway: A promising pharmacological approach to prevent endothelial dysfunction in cardiovascular diseases? Pharmacol Ther 131: $1-17,2011$.

19. Chiamvimonvat N, Ho CM, Tsai HJ and Hammock BD: The soluble epoxide hydrolase as a pharmaceutical target for hypertension. J Cardiovasc Pharmacol 50: 225-237, 2007.
20. Oguro A, Fujita N and Imaoka S: Regulation of soluble epoxide hydrolase $(\mathrm{sEH})$ in mice with diabetes: High glucose suppresses sEH expression. Drug Metab Pharmacokinet 24: 438-445, 2009.

21. Chaudhary KR, Abukhashim M, Hwang SH, Hammock BD and Seubert JM: Inhibition of soluble epoxide hydrolase by trans4-[4-(3-adamantan-1-yl-ureido)-cyclohexyloxy]-benzoic acid is protective against ischemia-reperfusion injury. J Cardiovasc Pharmacol 55: 67-73, 2010.

22. Qiu H, Li N, Liu JY, Harris TR, Hammock BD and Chiamvimonvat N: Soluble epoxide hydrolase inhibitors and heart failure. Cardiovasc Ther 29: 99-111, 2011.

23. Xu D, Li N, He Y, Timofeyev V, Lu L, Tsai HJ, Kim IH, Tuteja D, Mateo RK, Singapuri A, et al: Prevention and reversal of cardiac hypertrophy by soluble epoxide hydrolase inhibitors. Proc Natl Acad Sci USA 103: 18733-18738, 2006.

24. Imig JD and Hammock BD: Soluble epoxide hydrolase as a therapeutic target for cardiovascular diseases. Nat Rev Drug Discov 8: 794-805, 2009.

25. Lehman TJ, Walker SM, Mahnovski V and McCurdy D: Coronary arteritis in mice following the systemic injection of group B Lactobacillus casei cell walls in aqueous suspension. Arthritis Rheum 28: 652-659, 1985.

26. Noguchi S, Saito J, Kudo T, Hashiba E and Hirota K: Safety and efficacy of plasma exchange therapy for Kawasaki disease in children in intensive care unit: Case series. JA Clin Rep 4: 25, 2018.

27. Lee Y, Schulte DJ, Shimada K, Chen S, Crother TR, Chiba N, Fishbein MC, Lehman TJ and Arditi M: Interleukin-1 $\beta$ is crucial for the induction of coronary artery inflammation in a mouse model of Kawasaki disease. Circulation 125: 1542-1550, 2012.

28. Node K, Huo Y, Ruan X, Yang B, Spiecker M, Ley K, Zeldin DC and Liao JK: Anti-inflammatory properties of cytochrome P450 epoxygenase-derived eicosanoids. Science 285: 1276-1279, 1999.

29. Cai Z, Zhao G, Yan J, Liu W, Feng W, Ma B, Yang L, Wang JA, Tu L and Wang DW: CYP2J2 overexpression increases EETs and protects against angiotensin II-induced abdominal aortic aneurysm in mice. J Lipid Res 54: 1448-1456, 2013.

30. Zhao G, Tu L, Li X, Yang S, Chen C, Xu X, Wang P and Wang DW: Delivery of AAV2-CYP2J2 protects remnant kidney in the 5/6-nephrectomized rat via inhibition of apoptosis and fibrosis. Hum Gene Ther 23: 688-699, 2012.

31. Zhao G, Wang J, Xu X, Jing Y, Tu L, Li X, Chen C, Cianflone K, Wang P, Dackor RT, et al: Epoxyeicosatrienoic acids protect rat hearts against tumor necrosis factor- $\alpha$-induced injury. J Lipid Res 53: 456-466, 2012.

32. Chen W, Yang S, Ping W, Fu X, Xu Q and Wang J: CYP2J2 and EETs protect against lung ischemia/reperfusion injury via anti-inflammatory effects in vivo and in vitro. Cell Physiol Biochem 35: 2043-2054, 2015.

33. Spector AA: Arachidonic acid cytochrome P450 epoxygenase pathway. J Lipid Res 50 (Suppl): S52-S56, 2009.

34. Larsen BT, Gutterman DD and Hatoum OA: Emerging role of epoxyeicosatrienoic acids in coronary vascular function. Eur J Clin Invest 36: 293-300, 2006.

35. Gao F, Si F, Feng S, Yi Q and Liu R: Resistin enhances inflammatory cytokine production in coronary artery tissues by activating the NF- $\mathrm{KB}$ signaling. Biomed Res Int 2016: 3296437, 2016.

36. Hui-Yuen JS, Duong TT and Yeung RS: TNF-alpha is necessary for induction of coronary artery inflammation and aneurysm formation in an animal model of Kawasaki disease. J Immunol 176: 6294-6301, 2006.

37. Lau AC, Duong TT, Ito S and Yeung RS: Matrix metalloproteinase 9 activity leads to elastin breakdown in an animal model of Kawasaki disease. Arthritis Rheum 58: 854-863, 2008

38. Lau AC, Duong TT, Ito S, Wilson GJ and Yeung RS: Inhibition of matrix metalloproteinase-9 activity improves coronary outcome in an animal model of Kawasaki disease. Clin Exp Immunol 157: 300-309, 2009.

39. Maury CP, Salo E and Pelkonen P: Circulating interleukin-1 beta in patients with Kawasaki disease. N Engl J Med 319: 1670-1671, 1988.

40. Michaelis UR, Fisslthaler B, Barbosa-Sicard E, Falck JR, Fleming I and Busse R: Cytochrome P450 epoxygenases $2 \mathrm{C} 8$ and 2C9 are implicated in hypoxia-induced endothelial cell migration and angiogenesis. J Cell Sci 118: 5489-5498, 2005.

41. Cheranov SY, Karpurapu M, Wang D, Zhang B, Venema RC and Rao GN: An essential role for SRC-activated STAT-3 in 14,15-EET-induced VEGF expression and angiogenesis. Blood 111: 5581-5591, 2008. 
42. Potente M, Michaelis UR, Fisslthaler B, Busse R and Fleming I: Cytochrome P450 2C9-induced endothelial cell proliferation involves induction of mitogen-activated protein (MAP) kinase phosphatase-1, inhibition of the c-Jun N-terminal kinase, and up-regulation of cyclin D1. J Biol Chem 277: 15671-15676, 2002.

43. Kireeva ML, Mo FE, Yang GP and Lau LF: Cyr61, a product of a growth factor-inducible immediate-early gene, promotes cell proliferation, migration, and adhesion. Mol Cell Biol 16: 1326-1334, 1996.

44. Deng Y, Theken KN and Lee CR: Cytochrome P450 epoxygenases, soluble epoxide hydrolase, and the regulation of cardiovascular inflammation. J Mol Cell Cardiol 48: 331-341, 2010.
45. DeleriveP,FruchartJCandStaels B:Peroxisomeproliferator-activated receptors in inflammation control. J Endocrinol 169: 453-459, 2001.

46. Liu Y, Zhang Y, Schmelzer K, Lee TS, Fang X, Zhu Y, Spector AA, Gill S, Morisseau C, Hammock BD and Shyy JY: The antiinflammatory effect of laminar flow: The role of PPARgamma, epoxyeicosatrienoic acids, and soluble epoxide hydrolase. Proc Natl Acad Sci USA 102: 16747-16752, 2005.

c) (i) (9) This work is licensed under a Creative Commons Attribution-NonCommercial-NoDerivatives 4.0 International (CC BY-NC-ND 4.0) License. 